\title{
Implicit memory of dynamic information
}

\author{
LARS-GÖRAN NILSSON, ULRICH OLOFSSON, and LARS NYBERG \\ University of Umeá, Umeá, Sweden
}

\begin{abstract}
This paper describes an experiment designed to explore whether perceptual priming could be demonstrated for dynamic information. A patch-light technique with luminous spots on the main joints of the human body was used for presenting natural and distorted actions for subjects in a study trial, a recognition test, and an action-decision test. In the recognition test, the subjects were asked to identify those actions presented in the study trial. In the action-decision test, they were asked to decide whether or not the information shown depicted natural actions. The results showed significant priming effects for real actions but not for distorted actions. Contingency analyses revealed statistical independence between action-decision performance and recognition performance. It was concluded that perceptual priming of dynamic information is possible, given compatibility between the information presented and the specific characteristics of the perceptual system.
\end{abstract}

Recent years have seen a steady increase in the number of studies designed to explore the nature of implicit memory and its relationship to explicit memory. As specified by Graf and Schacter (1985), these two forms of memory differ in the sense that explicit memory requires a conscious recollection of the study episode as in standard recall or recognition tasks, whereas implicit memory as measured by word-stem and word-fragment completion, lexical decision, or word identification does not require such conscious recollection of a previous experience.

A common feature in most implicit-memory experiments to date is the verbal nature of the study and test materials. Only a few published studies have gone beyond the verbal domain in seeking to understand this form of memory. One such exception is a recent study by Schacter, Cooper, and Delaney (1990), in which subjects were presented with line drawings of possible and impossible objects. Possible objects were defined as familiar structures that could exist in the three-dimensional world, whereas impossible objects were defined as structures that are not possible to realize in three dimensions because of subtle surface, edge, or contour violations of possible objects. The overall rationale for using such materials was that the mental representation of objects is fundamental to an understanding of both perception and memory. By focusing on both perception and memory, it was assumed that more basic processes would be uncovered than had been possible in previous research with verbal materials.

The particular task Schacter et al. (1990) designed for assessing implicit memory was to have subjects classify previously seen or unseen line drawings of objects as possible or impossible in the three-dimensional world. In contrast to the recognition test used by Schacter et al. to assess

This research was supported by a grant from the Swedish Council for Research in the Humanities and Social Sciences. We are grateful to Sverker Runeson for valuable comments. Address correspondence to Lars-Göran Nilsson, Department of Psychology, University of Umeå, S-901 87 Umeå, Sweden. explicit memory, this object-decision task did not make explicit reference to, or require conscious recollection of, any specific previous encounter with a presented object.

The main findings in the Schacter et al. (1990) study were (a) significant priming for possible objects after encoding of global three-dimensional object structure, (b) no priming for impossible objects after encoding of twodimensional object features, (c) no difference between global versus local study processing for explicit memory, and (d) stochastic independence between object-decision and recognition performance. Schacter et al. (1990) interpreted these results to mean that implicit memory, but not explicit memory, depends on encoding of and access to structural descriptions of objects and that the structuraldescription system for objects is one example of a perceptual-representation system (Tulving \& Schacter, 1990) that is critical for implicit memory.

The purpose of the experiment reported here was to extend the implicit-memory phenomenon to still another domain, which should tap even more basic perceptual processing than that involved in the processing of static twoand three-dimensional objects. The domain in question is that of the dynamic information involved in movements and actions. Runeson (1977b; Runeson \& Frykholm, 1983) demonstrated that the perceptual system is especially well equipped to process such information and proposed "smart mechanisms" to be responsible for this efficiency. On the basis of such research within the realm of direct perception (e.g., Johansson, 1973; Runeson, 1977a; Runeson \& Frykholm, 1983; Turvey \& Shaw, 1979) and on the basis of the paradigm used by Schacter et al. (1990), a task was designed to assess implicit memory of dynamic information. We will refer to this task as the actiondecision task.

The stimulus materials used in this task were based on a patch-light technique developed by Johansson (1973), in which models performed various actions, visible only by luminous spots on the main joints of the body. Using such a technique, we prepared one set of actions per- 
formed by a model (e.g., jumping, walking, sitting up) to be used for a study trial, and from this set another two sets to be used at two subsequent test trials. The first of these was a recognition test, in which subjects were asked to indicate for each action shown whether they remembered having seen the action in the study trial. The second test was the action-decision task, in which subjects were asked to indicate for each action shown whether it was a natural or an unnatural action. Half the actions shown in this test were real actions performed by a model; the other half of the actions constituted real actions that had been distorted by having each light patch displaced to the left or the right from its original position.

This technique is useful for our purposes since it provides a method for isolating the contribution of kinematic information in motion (Runeson \& Frykholm, 1981). The science of mechanics distinguishes kinematics (i.e., motion as such) from dynamics, which is motion explained in terms of its causes and constraints. Runeson and Frykholm (1983) demonstrated in a long series of experiments that kinematic information can specify the dynamic components of actions. It is this analysis of kinematic and dynamic factors that underlie our claim that the patch-light technique is a useful method to tap basic processes of perception and memory.

On the basis of Schacter et al. (1990), it was predicted that significant priming would occur in the action-decision task and that an independent relationship in performance would be demonstrated between the explicit (recognition) and the implicit (action-decision) tasks. Moreover, it was expected that the orienting-task manipulation in the study trial would have no effect on priming but that a deep encoding would produce a higher recognition performance than a shallow encoding.

\section{METHOD}

\section{Subjects}

Subjects were 24 individually tested high school students. The subjects were paid an equivalent of $\$ 9$ for their participation.

\begin{abstract}
Apparatus
The actions were recorded with MacReflex (QUALISYS AB, Partille, Sweden). This is a system that, by emitting infrared light and registering its reflection from special markers, is capable of determining the positions in space of the markers. The MacReflex system consists of a video camera equipped with an infrared flash, a centroid processor that calculates the position of the markers in a plane, and Macintosh software for recording, editing, and displaying the sampled information. A Macintosh SE/30 was used for recording movements and for presenting the action patterns to the subjects being tested.
\end{abstract}

\section{Materials}

A program written by one of the authors was used to display the sampled data (i.e., the coordinates of the actor's joints) as black dots on the white Macintosh screen. One frame was displayed in each screen refresh, and since the sample rate of the MacReflex system is $50 \mathrm{~Hz}$ and the refresh rate of the SE/30 is $60.1 \mathrm{~Hz}$, the actions unfolded slightly faster (about $20 \%$ ) on the screen than when recorded. ${ }^{1}$

Thirty-two actions were adapted for the experiment. Each action sequence lasted $5 \mathrm{sec}$. The actions were of various kinds that could be completed within the 5-sec time limit, for example, jumping, kicking, leaping, etc. The actions were shown in either the same form as they had been recorded (real actions) or in a distorted form. The distortion was accomplished by offsetting the dots (joints) with \pm 50 pixels horizontal from the true position. The height of the actor on the screen when standing upright (the difference between the marker attached to the head and the one attached to the ankle) was about 200 pixels, so the distortion can be considered substantial.

A pretest was carried out to assess the consensus in classifying real and distorted actions. The subjects were shown the action sequences for $5 \mathrm{sec}$ and instructed to classify them as natural or unnatural (note that Schacter et al. allowed unlimited time for their pretest). The subjects classified $85 \%(.75-1.00)$ of the real actions and $74 \%(.69-.88)$ of the distorted actions correctly.

An additional pretest was done to determine a proper exposure time for the actions in the implicit test. The overall baseline performance was .67 when the actions were shown for $2 \mathrm{sec}$ (including the critical part of each action). This level was regarded as adequate, leaving room for priming effects to be detected. Subjects in the pretests were different from those in the main experiment.

\section{Design}

Three factors were manipulated in the experiment: orienting task, type of movement, and type of test. Half of the subjects were assigned to a shallow encoding condition and the other half to a deep encoding condition. Half of the actions presented to each subject were real actions and half were distorted actions. All subjects were tested with an implicit "action-decision test" and half of them were given a standard recognition test prior to the action-decision test.

\section{Procedure}

The subjects were presented in the study trial with 16 actions in sequence; each sequence was $5 \mathrm{sec}$ long. Eight of these sequences were real actions and 8 were distorted. The order of presentation was randomly determined for each subject. The subjects in the deep encoding condition were told that a series of movements would appear on the computer screen for $5 \mathrm{sec}$. They were instructed to try to identify and label each movement by stating what they thought the movement depicted. Similar instructions were given in the shallow encoding condition, but the subjects in this condition were instructed to state the general direction of each movement (left, right, up, or down).

Immediately after the study trial, half of the subjects (equally many from each study condition) were given a recognition test. They were presented with the 16 studied actions mixed with 16 new actions and were required, for each movement, to decide whether it had occurred in the study list or not.

Finally, all subjects were given the action-decision task. The same list as in the recognition test was presented (16 targets and 16 distractors). The action sequences were, however, reduced to the $2 \mathrm{sec}$ in the middle portion of the sequence (with the restriction that the critical part of each action should be included). The task was to decide whether these reduced sequences depicted possible natural actions or not. To avoid response bias, half of the subjects in this condition were instructed to detect natural actions and the rest of the subjects were instructed to detect impossible actions.

\section{RESULTS}

The results of the action-decision and recognition tests are first considered separately and then followed by a contingency analysis of the relation between them.

\section{Action Decision}

As can be seen in Table 1, there was a substantial priming effect in that a prior exposure to the actions facilitated performance on the decision task. The priming effect was statistically significant both for the deep encoding condition $[t(11)=3.21, p<.01]$ and the shallow encoding condition $[t(11)=3.0, p<.05]$. The table also shows that the priming effect is restricted to real actions; there is no priming for distorted actions. This finding 
Table 1

Proportions Correct on Recognition and Priming on Action Decision as a Function of Type of Movement and Study Task

\begin{tabular}{cccccr}
\hline & \multicolumn{2}{c}{ Undistorted } & & \multicolumn{2}{c}{ Distorted } \\
\cline { 2 - 3 } \cline { 5 - 6 } Study Task & RN & AD & & RN & AD \\
\hline Direction & .68 & .10 & & .46 & .04 \\
Label & .79 & .11 & & .63 & -.02 \\
\hline
\end{tabular}

Note-RN = recognition ([hits - false alarms]/[1 - false alarms]); $\mathrm{AD}=$ action decision (olds - news).

resembles the results obtained by Schacter et al. (1990), who reported priming for drawings of "possible" objects but not for "impossible" objects. A third effect to observe in the results presented in Table 1 is that the type of study task did not differentially affect performance on the action-decision task.

\section{Recognition}

An inspection of the recognition data in Table 1 suggests a superiority in recognition performance after deep encoding for both normal and distorted actions. This superiority was, however, not significant in either of the conditions $(p>.10)$. There was also a tendency to a higher recognition performance for real actions as compared with distorted actions $[t(11)=1.81, p<.10]$.

\section{Contingency Analysis of Action-Decision and Recognition Performance}

On the basis of previous research on the explicit-implicit memory distinction, the overall purpose of the contingency analysis was to determine whether action-decision performance is dependent on, or independent of, recognition memory. A significant priming effect was obtained only for real actions, and for this reason the contingency analysis is restricted to this material. The score of each subject on each item in each test was entered into a $2 \times 2$ contingency table with each of the four cells corresponding to one of the possible joint outcomes for studied items on the two tests. Yule's $Q$ was used for estimating the association between the tests, and a chi-square test suggested by Hayman and Tulving (1989) was used for evaluating the statistical significance of the association obtained. The results showed a $Q$ value of .20 and $\chi^{2}$ value of .31 , indicating lack of association between recognition-memory performance and action-decision performance.

\section{DISCUSSION}

This experiment demonstrated that priming of natural human actions is possible, in contrast to unnatural distorted actions, which did not show any priming. We conceive of this as a replication and an extension of the results obtained by Schacter et al. (1990). It is a replication in the sense that Schacter et al. found priming for possible objects but not for impossible objects. It is an extension of these results in the sense that priming appears to hold not only for perception of static objects but also for perception of dynamic actions. Although it would seem adequate to argue that the perception of dynamic actions requires more basic processing than does the perception of static objects, it is beyond the scope of this paper to try to prove this point. Suffice it to say that both these types of priming should be of a more fundamental nature than is perceptual priming in a verbal implicit test such as word-fragment completion.

The results obtained here also replicate those of Schacter et al. in the sense that the performance in the recognition task was found to be stochastically independent of the performance in the action-decision task. On the basis of the same reasoning used by Schacter et al., we regard the finding of stochastic independence as a suggestive clue that actiondecision and recognition performance depend on different underlying processes or separate memory systems.

In a recent paper, Kersteen-Tucker (1991) presented data that are strikingly similar to those obtained here and those obtained by Schacter et al. (1990). Kersteen-Tucker presented symmetrical and nonsymmetrical polygons for subjects in a lexical decision task and found long-term repetition priming for symmetrical polygons but not for nonsymmetrical ones. Thus, the fact that priming is demonstrated for possible objects, natural actions, and symmetrical polygons, but not for impossible objects, unnatural distorted actions, and nonsymmetrical polygons, might suggest that familiarity with the stimulus is a necessary requirement for priming to occur. Expressed differently, it might be argued that priming is a basic, taskand object-specific perceptual skill, which is activated by study and which is functioning properly only if the information presented is compatible with the specific mechanisms evolved in the perceptual system.

\section{REFERENCES}

Graf, P., \&Chacter, D. L. (1985). Implicit and explicit memory for new associations in normal and amnesic subjects. Journal of Experimental Psychology: Learning, Memory, \& Cognition, 11, 501-518.

Hayman, C. A. G., Tulving, E. (1989). Contingent dissociation between recognition and fragment completion: The method of triangulation. Journal of Experimental Psychology: Learning, Memory, \& Cognition, 15, 228-240.

Johansson, G. (1973). Visual perception of biological motion and a model for its analysis. Perception \& Psychophysics, 14, 201-211.

KERSTEEN-TUCKER, Z. (1991). Long-term repetition priming with symmetrical polygons and words. Memory \& Cognition, 19, 37-43.

RUNESON, S. (1977a). On the possibility of "smart" perceptual mechanisms. Scandinavian Journal of Psychology, 18, 172-179.

RUNESON, S. (1977b). On visual perception of dynamic events. Unpublished doctoral dissertation, Uppsala University, Uppsala, Sweden.

Runeson, S., \& Frykholm, G. (1981). Visual perception of lifted weight. Journal of Experimental Psychology: Human Perception \& Performance, 7, 733-740.

Runeson, S., \& Frykholm, G. (1983). Kinematic specification of dynamics as an informational basis for person-and-action perception: Expectation, gender recognition, and deceptive intention. Journal of Experimental Psychology: General, 112, 585-615.

Schacter, D. L., CoOper, L. A., \& Delaney, S. M. (1990). Implicit memory for unfamiliar objects depends on access to structural descriptions. Journal of Experimental Psychology: Learning, Memory, \& Cognition, 16, 5-24.

Tulving, E., SChacter, D. L. (1990). Priming and human memory systems. Science, 247, 301-306.

TURVEY, M. T., Shaw, R. (1979). The primacy of perceiving: An ecological reformulation of perception for understanding memory. In L.-G. Nilsson (Ed.), Perspectives on memory research (pp. 167-222). Hillsdale, NJ: Erlbaum.

\section{NOTE}

1. Runeson (personal communication) remarked that an artificial change in the speed of the movement disrupts the dynamic information, and it is possible that this has reduced the difference between our "real" and distorted actions.

(Manuscript received December 31, 1991.) 